\title{
Geometry of multilinear forms on $l_{1}$
}

\author{
Sung Guen KIM
}

\begin{abstract}
We characterize extreme, exposed and smooth points in the Banach space $\mathcal{L}\left({ }^{n} E\right)$ of continuous $n$-linear forms on $E$, and in its subspace $\mathcal{L}_{s}\left({ }^{n} E\right)$ of symmetric $n$-linear forms on $E$ when $E=l_{1}$ and $E=l_{1}^{m}$ for $n, m \in \mathbb{N}$ with $n, m \geq 2$.
\end{abstract}

\section{Introduction}

Throughout the paper, we let $n, m \in \mathbb{N}, n, m \geq 2$. We write $B_{E}$ for the closed unit ball of a real Banach space $E$, and the dual space of $E$ is denoted by $E^{*}$. An element $x \in B_{E}$ is called an extreme point of $B_{E}$ if $y, z \in B_{E}$ with $x=\frac{1}{2}(y+z)$ implies $x=y=z$. An element $x \in B_{E}$ is called an exposed point of $B_{E}$ if there is $f \in E^{*}$ such that $f(x)=1=\|f\|$ and $f(y)<1$ for every $y \in B_{E} \backslash\{x\}$. It is easy to see that every exposed point of $B_{E}$ is an extreme point. An element $x \in B_{E}$ is called a smooth point of $B_{E}$ if there is a unique $f \in E^{*}$ so that $f(x)=1=\|f\|$. We denote by $\operatorname{ext} B_{E}, \exp B_{E}$ and $\operatorname{sm} B_{E}$ the set of extreme points, the set of exposed points and the set of smooth points of $B_{E}$, respectively. A mapping $P: E \rightarrow \mathbb{R}$ is a continuous $n$-homogeneous polynomial if there exists a continuous $n$-linear form $T$ on the product $E \times \cdots \times E$ such that $P(x)=T(x, \ldots, x)$ for every $x \in E$. We denote by $\mathcal{P}\left({ }^{n} E\right)$ the Banach space of all continuous $n$-homogeneous polynomials from $E$ into $\mathbb{R}$ endowed with the norm $\|P\|=\sup _{\|x\|=1}|P(x)|$. We denote by $\mathcal{L}\left({ }^{n} E\right)$ the Banach space of all continuous $n$-linear forms on $E$ endowed with the norm $\|T\|=\sup _{\left\|x_{k}\right\|=1}\left|T\left(x_{1}, \ldots, x_{n}\right)\right|$ and $\mathcal{L}_{s}\left({ }^{n} E\right)$ denotes the closed subspace of all continuous symmetric $n$-linear forms on $E$. Notice that $\mathcal{L}\left({ }^{n} E\right)$ is identified with the dual of $n$-fold projective tensor product $\hat{\bigotimes}_{\pi, n} E$. With this identification, the action of a continuous $n$-linear form $T$ as a bounded linear functional on $\hat{\bigotimes}_{\pi, n} E$ is given by

Received August 15, 2020.

2020 Mathematics Subject Classification. 46A22.

Key words and phrases. Extreme points, exposed points and smooth points. https://doi.org/10.12697/ACUTM.2021.25.04 


$$
\left\langle\sum_{i=1}^{k} x^{(1), i} \otimes \cdots \otimes x^{(n), i}, T\right\rangle=\sum_{i=1}^{k} T\left(x^{(1), i}, \ldots, x^{(n), i}\right) .
$$

Notice also that $\mathcal{L}_{s}\left({ }^{n} E\right)$ is identified with the dual of $n$-fold symmetric projective tensor product $\hat{\bigotimes}_{s, \pi, n} E$. With this identification, the action of a continuous symmetric $n$-linear form $T$ as a bounded linear functional on $\hat{\bigotimes}_{s, \pi, n} E$ is given by

$$
\left\langle\sum_{i=1}^{k} \frac{1}{n !}\left(\sum_{\sigma} x^{\sigma(1), i} \otimes \cdots \otimes x^{\sigma(n), i}\right), T\right\rangle=\sum_{i=1}^{k} T\left(x^{(1), i}, \ldots, x^{(n), i}\right),
$$

where $\sigma$ goes over all permutations on $\{1, \ldots, n\}$. For more details about the theory of polynomials and multilinear mappings on Banach spaces, we refer to $[6]$.

Let us say a little bit about the history of classification problems of the extreme points, the exposed points, and the smooth points of the unit ball of continuous $n$-homogeneous polynomials on a Banach space.

We let $l_{p}^{n}=\mathbb{R}^{n}$ for every $1 \leq p \leq \infty$ equipped with the $l_{p}$-norm. Choi et al. ([2], [3], [4]) classified ext $B_{\mathcal{P}\left({ }^{2} l_{p}^{2}\right)}$ and $\operatorname{sm} B_{\mathcal{P}\left({ }^{2} l_{p}^{2}\right)}$ for $p=1,2$. Choi and Kim [5] classified $\operatorname{sm} B_{\mathcal{P}\left({ }^{2} l_{1}\right)}$. Grecu [7] classified the sets ext $B_{\mathcal{P}\left({ }^{2} l_{p}^{2}\right)}$ for $1<p<2$ or $2<p<\infty$. Kim et al. [26] showed that if $E$ is a separable real Hilbert space with $\operatorname{dim}(E) \geq 2$, then, $\operatorname{ext} B_{\mathcal{P}\left({ }^{2} E\right)}=\exp B_{\mathcal{P}\left({ }^{2} E\right)}$. Kim [8] classified $\exp B_{\mathcal{P}\left({ }^{2} l_{p}^{2}\right)}$ for $1 \leq p \leq \infty$. Kim ([10], [12]) characterized ext $B_{\mathcal{P}\left({ }^{2} d_{*}(1, w)^{2}\right)}$ and $\operatorname{sm} B_{\mathcal{P}\left({ }^{2} d_{*}(1, w)^{2}\right)}$, where $d_{*}(1, w)^{2}=\mathbb{R}^{2}$ with an octagonal norm $\|(x, y)\|_{w}=\max \left\{|x|,|y|, \frac{|x|+|y|}{1+w}\right\}$ for $0<w<1$. Kim [16] classified $\exp B_{\mathcal{P}\left({ }^{2} d_{*}(1, w)^{2}\right)}$ and showed that $\exp B_{\mathcal{P}\left({ }^{2} d_{*}(1, w)^{2}\right)} \neq \operatorname{ext} B_{\mathcal{P}\left({ }^{2} d_{*}(1, w)^{2}\right)}$. Recently, Kim ([17], [21]) classified ext $B_{\mathcal{P}\left(2 \mathbb{R}_{h\left(\frac{1}{2}\right)}^{2}\right)}$ and $\exp B_{\mathcal{P}\left(2 \mathbb{R}_{h\left(\frac{1}{2}\right)}^{2}\right)}$, where $\mathbb{R}_{h\left(\frac{1}{2}\right)}^{2}=\mathbb{R}^{2}$ with a hexagonal norm $\|(x, y)\|_{h\left(\frac{1}{2}\right)}=\max \left\{|y|,|x|+\frac{1}{2}|y|\right\}$.

Parallel to the classification problems of $\operatorname{ext} B_{\mathcal{P}\left({ }^{n} E\right)}$, $\exp B_{\mathcal{P}\left({ }^{n} E\right)}$, and $\operatorname{sm} B_{\mathcal{P}\left({ }^{n} E\right)}$ it seems to be very natural to study the, classification problems of extreme and exposed points of the unit ball of continuous (symmetric) multilinear forms on a Banach space.

Kim [9] classified $\operatorname{ext} B_{\mathcal{L}_{s}\left({ }^{2} l_{\infty}^{2}\right)}, \exp B_{\mathcal{L}_{s}\left({ }^{2} l_{\infty}^{2}\right)}$ and $\operatorname{sm} B_{\mathcal{L}_{s}\left(2 l_{\infty}^{2}\right)}$. It was shown that ext $B_{\mathcal{L}_{s}\left({ }^{2} l_{\infty}^{2}\right)}=\exp B_{\mathcal{L}_{s}\left({ }^{2} l_{\infty}^{2}\right)}$. Kim ([11], [13], [14], [15]) classified ext $B_{X}$ and $\exp B_{X}$, where $X=\mathcal{L}\left({ }^{2} d_{*}(1, w)^{2}\right)$ or $\mathcal{L}_{s}\left({ }^{2} d_{*}(1, w)^{2}\right)$. Kim ([18], [19]) also classified ext $B_{\mathcal{L}_{s}\left({ }^{2} l_{\infty}^{3}\right)}$, $\operatorname{ext} B_{\mathcal{L}_{s}\left({ }^{3} l_{\infty}^{2}\right)}$ and $\operatorname{sm} B_{\mathcal{L}_{s}\left({ }^{3} l_{\infty}^{2}\right)}$. It was shown that $\operatorname{ext} B_{\mathcal{L}_{s}\left({ }^{2} l_{\infty}^{3}\right)}=\exp B_{\mathcal{L}_{s}\left({ }^{2} l_{\infty}^{3}\right)}$ and $\operatorname{ext} B_{\mathcal{L}_{s}\left({ }^{3} l_{\infty}^{2}\right)}=\exp B_{\mathcal{L}_{s}\left({ }^{3} l_{\infty}^{2}\right)}$. Kim [20] characterized ${ }^{\infty} \operatorname{ext} B_{\mathcal{L}\left({ }^{2} l_{\infty}^{n}\right)}$ and $\operatorname{ext} B_{\mathcal{L}_{s}\left({ }^{2} l_{\infty}^{n}\right)}$, and showed that $\exp B_{\mathcal{L}\left({ }^{2} l_{\infty}^{n}\right)}=\operatorname{ext} B_{\mathcal{L}\left({ }^{2} l_{\infty}^{n}\right)}$ and $\exp B_{\mathcal{L}_{s}\left({ }^{2} l_{\infty}^{n}\right)}=\operatorname{ext} B_{\mathcal{L}_{s}\left({ }^{2} l_{\infty}^{n}\right)}$. Kim [22] characterized ext $B_{\mathcal{L}\left({ }^{2} l_{\infty}^{3}\right)}$ and $\exp B_{\mathcal{L}\left({ }^{2} l_{\infty}^{3}\right)}$. Kim [25] characterized $\operatorname{sm} B_{\mathcal{L}_{s}\left({ }^{n} l_{\infty}^{2}\right)}$. 
Kim [24] studied ext $B_{\mathcal{L}\left({ }^{2} l_{\infty}\right)}$. Cavalcante et al. [1] characterized ext $B_{\mathcal{L}\left({ }^{n} l_{\infty}^{m}\right)}$. Recently, Kim [23] classified ext $B_{\mathcal{L}\left({ }^{n} l_{\infty}^{2}\right)}$ and $\operatorname{ext} B_{\left.\mathcal{L}_{s}{ }^{n} l_{\infty}^{2}\right)}$. It was shown that $\left|\operatorname{ext} B_{\mathcal{L}\left({ }^{n} l_{\infty}^{2}\right)}\right|=2^{\left(2^{n}\right)}$ and $\left|\operatorname{ext} B_{\mathcal{L}_{s}\left({ }^{n} l_{\infty}^{2}\right)}\right|=2^{n+1}$, and that $\exp B_{\mathcal{L}\left({ }^{n} l_{\infty}^{2}\right)}=$ $\operatorname{ext} B_{\mathcal{L}\left({ }^{n} l_{\infty}^{2}\right)}$ and $\exp B_{\mathcal{L}_{s}\left({ }^{n} l_{\infty}^{2}\right)}=\operatorname{ext} B_{\mathcal{L}_{s}\left({ }^{n} l_{\infty}^{2}\right)}$.

In this paper, we characterize the extreme and exposed points of the unit balls of $\mathcal{L}\left({ }^{n} l_{1}\right)$ and $\mathcal{L}_{s}\left({ }^{n} l_{1}\right)$. We also characterize the smooth points of the unit balls of $\mathcal{L}\left({ }^{n} l_{1}^{m}\right)$ and $\mathcal{L}_{s}\left({ }^{n} l_{1}^{m}\right)$ for $m \geq 2$.

\section{The unit ball of $\mathcal{L}\left({ }^{n} l_{1}\right)$}

In this section we characterize $\operatorname{ext} B_{\mathcal{L}\left({ }^{n} l_{1}\right)}, \exp B_{\mathcal{L}\left({ }^{n} l_{1}\right)}$ and $\operatorname{sm} B_{\mathcal{L}\left({ }^{n} l_{1}^{m}\right)}$ for $n, m \geq 2$. First, we present an explicit formulae for the norm of $T \in \mathcal{L}\left({ }^{n} l_{1}\right)$.

Theorem 1. Let $T \in \mathcal{L}\left({ }^{n} l_{1}\right)$ with

$$
T\left(\left(x_{j}^{(1)}\right), \ldots,\left(x_{j}^{(n)}\right)\right)=\sum_{j_{1}, \ldots, j_{n} \in \mathbb{N}} a_{j_{1} \ldots j_{n}} x_{j_{1}}^{(1)} \ldots x_{j_{n}}^{(n)}
$$

for some $a_{j_{1} \ldots j_{n}} \in \mathbb{R}$. Then $\|T\|=\sup \left\{\left|a_{j_{1} \cdots j_{n}}\right|: j_{1}, \ldots, j_{n} \in \mathbb{N}\right\}$.

Proof. Note that, for $j_{1}, \ldots, j_{n} \in \mathbb{N}$, we have $\left|a_{j_{1} \ldots j_{n}}\right|=\left|T\left(e_{j_{1}}, \ldots, e_{j_{n}}\right)\right| \leq$ $\|T\|$. Hence,

$$
\|T\| \geq \sup \left\{\left|a_{j_{1} \ldots j_{n}}\right|: j_{1}, \ldots, j_{n} \in \mathbb{N}\right\} .
$$

On the other hand we get

$$
\begin{aligned}
\|T\|= & \sup \left\{\left|T\left(\left(x_{j}^{(1)}\right), \ldots,\left(x_{j}^{(n)}\right)\right)\right|:\left\|\left(x_{j}^{(k)}\right)\right\|_{1}=1, k=1, \ldots, n\right\} \\
\leq & \sup \left\{\sum_{j_{1}, \ldots, j_{n} \in \mathbb{N}}\left|a_{j_{1} \cdots j_{n}}\right|\left|x_{j_{1}}^{(1)}\right| \cdots\left|x_{j_{n}}^{(n)}\right|:\left\|\left(x_{j}^{(k)}\right)\right\|_{1}=1, k=1, \ldots, n\right\} \\
\leq & \sup \left\{\left|a_{j_{1} \cdots j_{n}}\right|: j_{1}, \ldots, j_{n} \in \mathbb{N}\right\} \\
& \times \sup \left\{\sum_{j \in \mathbb{N}}\left|x_{j}^{(1)}\right| \ldots \sum_{j \in \mathbb{N}}\left|x_{j}^{(n)}\right|:\left\|\left(x_{j}^{(k)}\right)\right\|_{1}=1, k=1, \ldots, n\right\} \\
= & \sup \left\{\left|a_{j_{1} \cdots j_{n}}\right|: j_{1}, \ldots, j_{n} \in \mathbb{N}\right\} .
\end{aligned}
$$

Now we characterize all extreme points of the unit ball of $\mathcal{L}\left({ }^{n} l_{1}\right)$.

Theorem 2. Let $T \in \mathcal{L}\left({ }^{n} l_{1}\right)$ be defined by (1) and let $\|T\|=1$. Then $T \in \operatorname{ext} B_{\mathcal{L}\left({ }^{n} l_{1}\right)}$ if and only if $\left|a_{j_{1} \cdots j_{n}}\right|=1$ for all $j_{1}, \ldots, j_{n} \in \mathbb{N}$.

Proof. By Theorem 1, $\left|a_{j_{1} \ldots j_{n}}\right| \leq 1$ for all $j_{1}, \ldots, j_{n} \in \mathbb{N}$. 
Necessity. Assume the contrary. Then there are $j_{1}^{\prime}, \ldots, j_{n}^{\prime} \in \mathbb{N}$ such that $\left|a_{j_{1}^{\prime} \cdots j_{n}^{\prime}}\right|<1$. Let $S_{j_{1}^{\prime} \cdots j_{n}^{\prime}} \in \mathcal{L}\left({ }^{n} l_{1}\right)$ be defined by

$$
S_{j_{1}^{\prime} \cdots j_{n}^{\prime}}\left(\left(x_{j}^{(1)}\right), \ldots,\left(x_{j}^{(n)}\right)\right)=x_{j_{1}^{\prime}}^{(1)} \cdots x_{j_{n}^{\prime}}^{(n)} .
$$

Choose $\epsilon_{0}>0$ such that $\left|a_{j_{1}^{\prime} \cdots j_{n}^{\prime}}\right|+\epsilon_{0}<1$ and set $R^{ \pm}=T \pm \epsilon_{0} S_{j_{1}^{\prime} \cdots j_{n}^{\prime}}$. By Theorem 1, we have $\left\|R^{ \pm}\right\|=1$. Since $T=\frac{1}{2}\left(R^{+}+R^{-}\right), T$ is not extreme. This is a contradiction. Hence, $\left|a_{j_{1} \cdots j_{n}}\right|=1$ for all $j_{1}, \ldots, j_{n} \in \mathbb{N}$.

Sufficiency. Suppose that $T=\frac{1}{2}\left(T_{1}+T_{2}\right)$ for some $T_{j} \in \mathcal{L}\left({ }^{n} l_{1}\right)$ with $\left\|T_{j}\right\|=1$. Write

$$
\begin{aligned}
& T_{1}\left(\left(x_{j}^{(1)}\right), \ldots,\left(x_{j}^{(n)}\right)\right)=\sum_{j_{1}, \ldots, j_{n} \in \mathbb{N}} b_{j_{1} \cdots j_{n}} x_{j_{1}}^{(1)} \cdots x_{j_{n}}^{(n)}, \\
& T_{2}\left(\left(x_{j}^{(1)}\right), \ldots,\left(x_{j}^{(n)}\right)\right)=\sum_{j_{1}, \ldots, j_{n} \in \mathbb{N}} c_{j_{1} \cdots j_{n}} x_{j_{1}}^{(1)} \cdots x_{j_{n}}^{(n)}
\end{aligned}
$$

for some $b_{j_{1} \cdots j_{n}}, c_{j_{1} \cdots j_{n}} \in \mathbb{R}$ with $\left|b_{j_{1} \cdots j_{n}}\right| \leq 1,\left|c_{j_{1} \cdots j_{n}}\right| \leq 1$ for all $j_{1}, \ldots, j_{n} \in$ $\mathbb{N}$. Then, $a_{j_{1} \cdots j_{n}}=\frac{1}{2}\left(b_{j_{1} \cdots j_{n}}+c_{j_{1} \cdots j_{n}}\right)$ for all $j_{1}, \ldots, j_{n} \in \mathbb{N}$. Since $\left|a_{j_{1} \cdots j_{n}}\right|=$ 1 for all $j_{1}, \ldots, j_{n} \in \mathbb{N}, a_{j_{1} \cdots j_{n}}=b_{j_{1} \cdots j_{n}}=c_{j_{1} \cdots j_{n}}$ for all $j_{1}, \ldots, j_{n} \in \mathbb{N}$. Therefore, $T=T_{j}$ for $j=1,2$. Hence $T \in \operatorname{ext} B_{\mathcal{L}\left({ }^{n} l_{1}\right)}$.

The following theorem shows that every extreme point of the unit ball of $\mathcal{L}\left({ }^{n} l_{1}\right)$ is exposed.

Theorem 3. The equality $\exp B_{\mathcal{L}\left({ }^{n} l_{1}\right)}=\operatorname{ext} B_{\mathcal{L}\left({ }^{n} l_{1}\right)}$ holds.

Proof. Let $T \in \operatorname{ext} B_{\mathcal{L}\left({ }^{n} l_{1}\right)}$. By Theorem 2, the equality (1) holds for some $a_{j_{1} \cdots j_{n}} \in \mathbb{R}$ with $\left|a_{j_{1} \cdots j_{n}}\right|=1$ for all $j_{1}, \ldots, j_{n} \in \mathbb{N}$. Let $\phi: \mathbb{N}^{n} \rightarrow \mathbb{N}$ be a bijection. Define $f \in \mathcal{L}\left({ }^{n} l_{1}\right)^{*}$ by

$$
f(S):=\sum_{\left(j_{1}, \ldots, j_{n}\right) \in \mathbb{N}^{n}} \frac{1}{2^{\phi\left(j_{1}, \ldots, j_{n}\right)}} \operatorname{sign}\left(a_{j_{1} \cdots j_{n}}\right) S\left(e_{j_{1}}, \ldots, e_{j_{n}}\right) .
$$

Then, by Theorem 2 ,

$$
f(T)=\sum_{\left(j_{1}, \ldots, j_{n}\right) \in \mathbb{N}^{n}} \frac{1}{2^{\phi\left(j_{1}, \ldots, j_{n}\right)}}\left|a_{j_{1} \cdots j_{n}}\right|=\sum_{\left(j_{1}, \ldots, j_{n}\right) \in \mathbb{N}^{n}} \frac{1}{2^{\phi\left(j_{1}, \ldots, j_{n}\right)}}=1 .
$$

It follows that

$$
\begin{aligned}
1 & =f(T) \leq\|f\| \\
& =\sup \left\{\sum_{\left(j_{1}, \ldots, j_{n}\right) \in \mathbb{N}^{n}} \frac{1}{2^{\phi\left(j_{1}, \ldots, j_{n}\right)}}\left|S\left(e_{j_{1}}, \ldots, e_{j_{n}}\right)\right|: S \in \mathcal{L}\left({ }^{n} l_{1}\right),\|S\|=1\right\} \\
& \leq \sum_{\left(j_{1}, \ldots, j_{n}\right) \in \mathbb{N}^{n}} \frac{1}{2^{\phi\left(j_{1}, \ldots, j_{n}\right)}}=1 .
\end{aligned}
$$


Hence $\|f\|=1$.

Claim: $f(S)<1$ for every $S \in B_{\mathcal{L}\left({ }^{n} l_{1}\right)}$ with $S \neq T$.

It is enough to show that if $f(S)=1$ for some $S \in B_{\mathcal{L}\left({ }^{n} l_{1}\right)}$, then $S=T$. We have

$$
\begin{aligned}
& \sum_{\left(j_{1}, \ldots, j_{n}\right) \in \mathbb{N}^{n}} \frac{1}{2^{\phi\left(j_{1}, \ldots, j_{n}\right)}}\left|a_{j_{1} \cdots j_{n}}\right|=1=f(S) \\
= & \sum_{\left(j_{1}, \ldots, j_{n}\right) \in \mathbb{N}^{n}} \frac{1}{2^{\phi\left(j_{1}, \ldots, j_{n}\right)}} \operatorname{sign}\left(a_{j_{1} \cdots j_{n}}\right) S\left(e_{j_{1}}, \ldots, e_{j_{n}}\right),
\end{aligned}
$$

which implies that

$$
S\left(e_{j_{1}}, \ldots, e_{j_{n}}\right)=a_{j_{1} \cdots j_{n}}=T\left(e_{j_{1}}, \ldots, e_{j_{n}}\right)
$$

for all $j_{1}, \ldots, j_{n} \in \mathbb{N}$. By the $n$-linearity, $S=T$. Therefore, $f$ exposes $T$. Hence $T \in \exp B_{\mathcal{L}\left({ }^{n} l_{1}\right)}$.

We characterize all smooth points of the unit ball of $\mathcal{L}\left({ }^{n} l_{1}^{m}\right)$ for $n, m \geq 2$.

Theorem 4. Let $n, m \geq 2$, and let $T \in \mathcal{L}\left({ }^{n} l_{1}^{m}\right)$ with $\|T\|=1$ and

$$
T\left(\left(x_{j}^{(1)}\right), \ldots,\left(x_{j}^{(n)}\right)\right)=\sum_{1 \leq j_{1}, \ldots, j_{n} \leq m} a_{j_{1} \cdots j_{n}} x_{j_{1}}^{(1)} \cdots x_{j_{n}}^{(n)} .
$$

Then $T \in \operatorname{sm} B_{\mathcal{L}\left({ }^{n} l_{1}^{m}\right)}$ if and only if there are $j_{1}^{\prime}, \ldots, j_{n}^{\prime} \in\{1, \ldots, m\}$ such that

$$
1=\left|a_{j_{1}^{\prime} \cdots j_{n}^{\prime}}\right|>\left|a_{j_{1} \cdots j_{n}}\right|
$$

for all $j_{1}, \ldots, j_{n} \in\{1, \ldots, m\}$ with $\left(j_{1}, \ldots, j_{n}\right) \neq\left(j_{1}^{\prime}, \ldots, j_{n}^{\prime}\right)$.

Proof. Sufficiency. Let $f \in \mathcal{L}\left({ }^{n} l_{1}^{m}\right)^{*}$ be such that $f(T)=1=\|f\|$. As $\mathcal{L}\left({ }^{n} l_{1}^{m}\right)^{*}=\otimes_{\pi, n} l_{1}^{m}$, there are $k \in \mathbb{N}$ and $f^{(1), i}, \ldots, f^{(n), i} \in l_{1}^{m}$ such that

$$
f=\sum_{i=1}^{k} f^{(1), i} \otimes \ldots \otimes f^{(n), i} .
$$

We claim that $f=\operatorname{sign}\left(a_{j_{1}^{\prime} \ldots j_{n}^{\prime}}\right)\left(e_{j_{1}^{\prime}} \otimes \ldots \otimes e_{j_{n}^{\prime}}\right)$. Indeed, let $j_{1}, \ldots, j_{n} \in$ $\{1, \ldots, m\}$ be such that $\left(j_{1}, \ldots, j_{n}\right) \neq\left(j_{1}^{\prime}, \ldots, j_{n}^{\prime}\right)$, and let $S_{j_{1} \cdots j_{n}} \in \mathcal{L}\left({ }^{n} l_{1}^{m}\right)$ be defined by

$$
S_{j_{1} \cdots j_{n}}\left(\left(x_{j}^{(1)}\right), \ldots,\left(x_{j}^{(n)}\right)\right)=x_{j_{1}}^{(1)} \cdots x_{j_{n}}^{(n)} .
$$

Choose $\epsilon_{0}>0$ such that $\left|a_{j_{1} \cdots j_{n}}\right|+\epsilon_{0}<1$ and set $R^{ \pm}=T \pm \epsilon_{0} S_{j_{1} \cdots j_{n}}$. By Theorem 1 we have $\left\|R^{ \pm}\right\|=1$. Thus,

$$
\begin{aligned}
1 & \geq \max \left\{\left|f\left(R^{ \pm}\right)\right|\right\}=\max \left\{\left|f(T) \pm \epsilon_{0} f\left(S_{j_{1} \cdots j_{n}}\right)\right|\right\} \\
& =|f(T)|+\epsilon_{0}\left|f\left(S_{j_{1} \cdots j_{n}}\right)\right|=1+\epsilon_{0}\left|f\left(S_{j_{1} \cdots j_{n}}\right)\right|,
\end{aligned}
$$


which implies that $f\left(S_{j_{1} \cdots j_{n}}\right)=0$ for all $j_{1}, \ldots, j_{n} \in\{1, \ldots, m\}$ such that $\left(j_{1}, \ldots, j_{n}\right) \neq\left(j_{1}^{\prime}, \ldots, j_{n}^{\prime}\right)$. Hence

$$
\left|a_{j_{1}^{\prime} \cdots j_{n}^{\prime}}\right|=1=f(T)=a_{j_{1}^{\prime} \cdots j_{n}^{\prime}} f\left(S_{j_{1}^{\prime} \cdots j_{n}^{\prime}}\right),
$$

which shows that $f\left(S_{j_{1}^{\prime} \cdots j_{n}^{\prime}}\right)=\operatorname{sign}\left(a_{j_{1}^{\prime} \cdots j_{n}^{\prime}}\right)$. Thus, for $S \in \mathcal{L}\left({ }^{n} l_{1}^{m}\right)$,

$$
\begin{aligned}
f(S) & =\sum_{1 \leq i_{1}, \ldots, i_{n} \leq m} S\left(e_{i_{1}}, \ldots, e_{i_{n}}\right) f\left(S_{j_{1} \cdots j_{n}}\right) \\
& =S\left(e_{j_{1}^{\prime}}, \ldots, e_{j_{n}^{\prime}}\right) f\left(S_{j_{1}^{\prime} \cdots j_{n}^{\prime}}\right)=S\left(e_{j_{1}^{\prime}}, \ldots, e_{j_{n}^{\prime}}\right) \operatorname{sign}\left(a_{j_{1}^{\prime} \cdots j_{n}^{\prime}}\right) \\
& =\operatorname{sign}\left(a_{j_{1}^{\prime} \cdots j_{n}^{\prime}}\right)\left(e_{j_{1}^{\prime}} \otimes \ldots \otimes e_{j_{n}^{\prime}}\right)(S) .
\end{aligned}
$$

Therefore, $f$ is uniquely determined and $T \in \operatorname{sm} B_{\mathcal{L}\left({ }^{n} l_{1}^{m}\right)}$.

Necessity. By Theorem 1,

$$
1=\|T\|=\max \left\{\left|a_{j_{1} \cdots j_{n}}\right|: 1 \leq j_{1}, \ldots, j_{n} \leq m\right\} .
$$

Hence there are $j_{1}^{\prime}, \ldots, j_{n}^{\prime} \in\{1, \ldots, m\}$ such that $1=\left|a_{j_{1}^{\prime} \cdots j_{n}^{\prime}}\right|$.

Claim: $\left|a_{j_{1} \cdots j_{n}}\right|<1$ for all $j_{1}, \ldots, j_{n} \in\{1, \ldots, m\}$ with $\left(j_{1}, \ldots, j_{n}\right) \neq$ $\left(j_{1}^{\prime}, \ldots, j_{n}^{\prime}\right)$.

If not, then there are $i_{1}^{\prime}, \ldots, i_{n}^{\prime} \in\{1, \ldots, m\}$ such that $\left(i_{1}^{\prime}, \ldots, i_{n}^{\prime}\right) \neq$ $\left(j_{1}^{\prime}, \ldots, j_{n}^{\prime}\right)$ and $1=\left|a_{i_{1}^{\prime} \cdots i_{n}^{\prime}}\right|$. Let $f_{1}, f_{2} \in \mathcal{L}\left({ }^{n} l_{1}^{m}\right)^{*}$ be defined by

$f_{1}=\operatorname{sign}\left(a_{j_{1}^{\prime} \cdots j_{n}^{\prime}}\right)\left(e_{j_{1}^{\prime}} \otimes \ldots \otimes e_{j_{n}^{\prime}}\right), \quad f_{2}=\operatorname{sign}\left(a_{i_{1}^{\prime} \cdots i_{n}^{\prime}}\right)\left(e_{i_{1}^{\prime}} \otimes \ldots \otimes e_{i_{n}^{\prime}}\right)$.

Then

$$
f_{1} \neq f_{2}, f_{j}(T)=1=\left\|f_{j}\right\|(j=1,2) .
$$

This is a contradiction. Therefore, we have proved the claim.

Theorem 5. Let $n \geq 2$ and let $T \in \mathcal{L}\left({ }^{n} l_{1}\right)$ be defined by (1) such that $\|T\|=1$. Suppose that $\left|a_{j_{1}^{\prime} \cdots j_{n}^{\prime}}\right|=1=\left|a_{i_{1}^{\prime} \cdots i_{n}^{\prime}}\right|$ for some $\left(j_{1}^{\prime}, \ldots, j_{n}^{\prime}\right) \neq$ $\left(i_{1}^{\prime}, \ldots, i_{n}^{\prime}\right) \in \mathbb{N}^{n}$. Then $T \notin \operatorname{sm} B_{\mathcal{L}\left({ }^{n} l_{1}\right)}$.

Proof. Let $f_{1}, f_{2} \in \mathcal{L}\left({ }^{n} l_{1}\right)^{*}$ be defined by (4). Then,

$$
f_{1} \neq f_{2}, f_{j}(T)=1=\left\|f_{j}\right\|(j=1,2) .
$$

Therefore, $T \notin \operatorname{sm} B_{\mathcal{L}\left({ }^{n} l_{1}\right)}$. 


\section{The unit ball of $\mathcal{L}_{s}\left({ }^{n} l_{1}\right)$}

In this section we characterize $\operatorname{ext} B_{\mathcal{L}_{s}\left({ }^{n} l_{1}\right)}, \exp B_{\mathcal{L}_{s}\left({ }^{n} l_{1}\right)}$ and $\operatorname{sm} B_{\mathcal{L}_{s}\left({ }^{n} l_{1}^{m}\right)}$ for $n, m \geq 2$. The first theorem characterizes all extreme points of the unit ball of $\mathcal{L}_{s}\left({ }^{n} l_{1}\right)$.

Theorem 6. Let $T \in \mathcal{L}_{s}\left({ }^{n} l_{1}\right)$ be defined by (1) and let $\|T\|=1$. Then $T \in \operatorname{ext} B_{\mathcal{L}_{s}\left({ }^{n} l_{1}\right)}$ if and only if $\left|a_{j_{1} \cdots j_{n}}\right|=1$ for all $j_{1}, \ldots, j_{n} \in \mathbb{N}$.

Proof. By Theorem 1, $\left|a_{j_{1} \ldots j_{n}}\right| \leq 1$ for all $j_{1}, \ldots, j_{n} \in \mathbb{N}$.

Sufficiency. Assume the contrary. There are $j_{1}^{\prime}, \ldots, j_{n}^{\prime} \in \mathbb{N}$ such that $\left|a_{j_{1}^{\prime} \cdots j_{n}^{\prime}}\right|<1$. Let $S_{j_{1}^{\prime} \cdots j_{n}^{\prime}} \in \mathcal{L}\left({ }^{n} l_{1}\right)$ be defined by $(2)$. Choose $\epsilon_{0}>0$ such that $\left|a_{j_{1}^{\prime} \cdots j_{n}^{\prime}},\right|+\epsilon_{0}<1$. Let $L^{ \pm} \in \mathcal{L}_{s}\left({ }^{n} l_{1}\right)$ be defined by

$$
L^{ \pm}=T \pm \frac{\epsilon_{0}}{n !} \sum_{\sigma} S_{j_{\sigma(1)}^{\prime} \cdots j_{\sigma(n)}^{\prime}},
$$

where $\sigma$ is a permutation on $\{1, \ldots, n\}$. By Theorem 1 we have $\left\|L^{ \pm}\right\|=1$. Since $T=\frac{1}{2}\left(L^{+}+L^{-}\right), T$ is not extreme. This is a contradiction. Hence, $\left|a_{j_{1} \cdots j_{n}}\right|=1$ for all $j_{1}, \ldots, j_{n} \in \mathbb{N}$.

The proof of necessity is identical to the proof of necessity of Theorem 2 .

We show that every extreme point of the unit ball of $\mathcal{L}_{s}\left({ }^{n} l_{1}\right)$ is exposed.

Theorem 7. The equality $\exp B_{\mathcal{L}_{s}\left({ }^{n} l_{1}\right)}=\operatorname{ext} B_{\mathcal{L}_{s}\left({ }^{n} l_{1}\right)}$ is true.

Proof. It is identical to that for Theorem 3.

The following theorem shows a relation between the spaces $\mathcal{L}\left({ }^{n} l_{1}\right)$ and $\mathcal{L}_{s}\left({ }^{n} l_{1}\right)$.

Theorem 8. Let $n, m \geq 2$. Then:

(a) $\operatorname{ext} B_{\mathcal{L}_{s}\left({ }^{n} l_{1}\right)}=\operatorname{ext} B_{\mathcal{L}\left({ }^{n} l_{1}\right)} \cap \mathcal{L}_{s}\left({ }^{n} l_{1}\right)$;

(b) $\exp B_{\mathcal{L}_{s}\left({ }^{n} l_{1}\right)}=\exp B_{\mathcal{L}\left({ }^{n} l_{1}\right)} \cap \mathcal{L}_{s}\left({ }^{n} l_{1}\right)$.

Proof. The statement (a) follows from Theorems 2 and 6, and (b) follows from Theorems 3 and 7 in view of (a).

We characterize all smooth points of the unit ball of $\mathcal{L}_{s}\left({ }^{n} l_{1}^{m}\right)$ for $n, m \geq 2$.

Theorem 9. For $n, m \geq 2$, let $T \in \mathcal{L}_{s}\left({ }^{n} l_{1}^{m}\right)$ be the same as in Theorem 4 . Then $T \in \operatorname{sm} B_{\mathcal{L}_{s}\left({ }^{n} l_{1}^{m}\right)}$ if and only if there are $j_{1}^{\prime}, \ldots, j_{n}^{\prime} \in\{1, \ldots, m\}$ such that

$$
1=\left|a_{j_{\sigma(1)}^{\prime} \cdots j_{\sigma(n)}^{\prime}}\right|>\left|a_{j_{1} \cdots j_{n}}\right|
$$

for all $j_{1}, \ldots, j_{n} \in\{1, \ldots, m\}$ with $\left(j_{1}, \ldots, j_{n}\right) \neq\left(j_{\sigma(1)}^{\prime}, \ldots, j_{\sigma(n)}^{\prime}\right)$, where $\sigma$ is a permutation on $\{1, \ldots, n\}$. 
Proof. Sufficiency. Let $f \in \mathcal{L}_{s}\left({ }^{n} l_{1}^{m}\right)^{*}$ be such that $f(T)=1=\|f\|$. We claim that

$$
f=\operatorname{sign}\left(a_{j_{1}^{\prime} \cdots j_{n}^{\prime}}\right) \frac{1}{n !} \sum_{\sigma}\left(e_{j_{\sigma(1)}^{\prime}} \otimes \ldots \otimes e_{j_{\sigma(n)}^{\prime}}\right) .
$$

Indeed, let $j_{1}, \ldots, j_{n} \in\{1, \ldots, m\}$ be such that $\left(j_{1}, \ldots, j_{n}\right) \neq\left(j_{\sigma(1)}^{\prime}, \ldots, j_{\sigma(n)}^{\prime}\right)$ for every permutation $\sigma$. Let $S_{j_{1} \cdots j_{n}} \in \mathcal{L}\left({ }^{n} l_{1}^{m}\right)$ be defined by (3). Choose $\epsilon_{0}>0$ such that $\left|a_{j_{1} \cdots j_{n}}\right|+\epsilon_{0}<1$. Let $L^{ \pm} \in \mathcal{L}_{s}\left({ }^{n} l_{1}\right)$ be defined by

$$
L^{ \pm}=T \pm \frac{\epsilon_{0}}{n !} \sum_{\sigma} S_{j_{\sigma(1)} \cdots j_{\sigma(n)}} .
$$

By Theorem 1, we have $\left\|L^{ \pm}\right\|=1$. Thus

$$
\begin{aligned}
1 & \geq \max \left\{\left|f\left(L^{ \pm}\right)\right|\right\}=\max \left\{\left|f(T) \pm \frac{\epsilon_{0}}{n !} f\left(\sum_{\sigma} S_{j_{\sigma(1)} \cdots j_{\sigma(n)}}\right)\right|\right\} \\
& =|f(T)|+\frac{\epsilon_{0}}{n !}\left|f\left(\sum_{\sigma} S_{j_{\sigma(1)} \cdots j_{\sigma(n)}}\right)\right|=1+\frac{\epsilon_{0}}{n !}\left|f\left(\sum_{\sigma} S_{j_{\sigma(1)} \cdots j_{\sigma(n)}}\right)\right|,
\end{aligned}
$$

which implies that $f\left(\sum_{\sigma} S_{j_{\sigma(1)} \cdots j_{\sigma(n)}}\right)=0$ for all $j_{1}, \ldots, j_{n} \in\{1, \ldots, m\}$ such that $\left(j_{1}, \ldots, j_{n}\right) \neq\left(j_{\sigma(1)}^{\prime}, \ldots, j_{\sigma(n)}^{\prime}\right)$ for every permutation $\sigma$. Hence,

$$
\left|a_{j_{1}^{\prime} \cdots j_{n}^{\prime}}\right|=1=f(T)=a_{j_{1}^{\prime} \cdots j_{n}^{\prime}} \frac{1}{n !} f\left(\sum_{\sigma} S_{j_{\sigma(1)}^{\prime} \cdots j_{\sigma(n)}^{\prime}}\right),
$$

which shows that $\frac{1}{n !} f\left(\sum_{\sigma} S_{j_{\sigma(1)}^{\prime} \cdots j_{\sigma(n)}^{\prime}}\right)=\operatorname{sign}\left(a_{j_{1}^{\prime} \cdots j_{n}^{\prime}}\right)$. Thus, for $S \in$ $\mathcal{L}\left({ }^{n} l_{1}^{m}\right)$,

$$
\begin{aligned}
& f(S)=\sum_{1 \leq i_{1}, \ldots, i_{n} \leq m} S\left(e_{i_{1}}, \ldots, e_{i_{n}}\right) \frac{1}{n !} f\left(\sum_{\sigma} S_{j_{\sigma(1)} \cdots j_{\sigma(n)}}\right) \\
& =S\left(e_{j_{1}^{\prime}}, \ldots, e_{j_{n}^{\prime}}\right) \frac{1}{n !} f\left(\sum_{\sigma} S_{j_{\sigma(1)}^{\prime} \cdots j_{\sigma(n)}^{\prime}}\right)=S\left(e_{j_{1}^{\prime}}, \ldots, e_{j_{n}^{\prime}}\right) \operatorname{sign}\left(a_{j_{1}^{\prime} \cdots j_{n}^{\prime}}\right) \\
& =\operatorname{sign}\left(a_{j_{1}^{\prime} \cdots j_{n}^{\prime}}\right) \frac{1}{n !} \sum_{\sigma}\left(e_{j_{\sigma(1)}^{\prime}} \otimes \ldots \otimes e_{j_{\sigma(n)}^{\prime}}\right)(S) .
\end{aligned}
$$

Therefore, $f$ is uniquely determined and $T \in \operatorname{sm} B_{\mathcal{L}\left({ }^{n} l_{1}^{m}\right)}$.

Necessary. By Theorem 1,

$$
1=\|T\|=\max \left\{\left|a_{j_{1} \cdots j_{n}}\right|: 1 \leq j_{1}, \ldots, j_{n} \leq m\right\} .
$$


Hence, there are $j_{1}^{\prime}, \ldots, j_{n}^{\prime} \in\{1, \ldots, m\}$ such that $1=\left|a_{j_{1}^{\prime} \cdots j_{n}^{\prime}}\right|$. Note that since $T$ is symmetric, $1=\left|a_{j_{\sigma(1)}^{\prime} \cdots j_{\sigma(n)}^{\prime}}\right|$ for every permutation $\sigma$ on $\{1, \ldots, n\}$.

Claim: $\left|a_{j_{1} \cdots j_{n}}\right|<1$ for all $j_{1}, \ldots, j_{n} \in\{1, \ldots, m\}$ such that $\left(j_{1}, \ldots, j_{n}\right) \neq$ $\left(j_{\sigma(1)}^{\prime}, \ldots, j_{\sigma(n)}^{\prime}\right)$ for every permutation $\sigma$ on $\{1, \ldots, n\}$.

If not, then there are $i_{1}^{\prime}, \ldots, i_{n}^{\prime} \in\{1, \ldots, m\}$ such that $1=\left|a_{i_{1}^{\prime} \cdots i_{n}^{\prime}}\right|$ and $\left(i_{1}^{\prime}, \ldots, i_{n}^{\prime}\right) \neq\left(j_{\sigma(1)}^{\prime}, \ldots, j_{\sigma(n)}^{\prime}\right)$ for every permutation $\sigma$ on $\{1, \ldots, n\}$. Let $f_{1}, f_{2} \in \mathcal{L}_{s}\left({ }^{n} l_{1}^{m}\right)^{*}$ be defined by

$$
\begin{aligned}
& f_{1}=\operatorname{sign}\left(a_{j^{\prime} \cdots j^{\prime}}\right) \frac{1}{n !} \sum_{\sigma}\left(e_{j_{\sigma(1)}^{\prime}} \otimes \ldots \otimes e_{j_{\sigma(n)}^{\prime}}\right), \\
& f_{2}=\operatorname{sign}\left(a_{i_{1}^{\prime} \cdots i_{n}^{\prime}}\right) \frac{1}{n !} \sum_{\sigma}\left(e_{i_{\sigma(1)}^{\prime}} \otimes \ldots \otimes e_{i_{\sigma(n)}^{\prime}}\right) .
\end{aligned}
$$

Then,

$$
f_{1} \neq f_{2}, f_{j}(T)=1=\left\|f_{j}\right\|(j=1,2) .
$$

This is a contradiction. Therefore, we have proved the claim.

Theorem 10. For $n \geq 2$, let $T \in \mathcal{L}_{s}\left({ }^{n} l_{1}\right)$ be the same as in Theorem 5. Suppose that $\left|a_{j_{1}^{\prime} \cdots j_{n}^{\prime}}\right|=1=\left|a_{i_{1}^{\prime} \cdots i_{n}^{\prime}}\right|$ for some $\left(j_{1}^{\prime}, \ldots, j_{n}^{\prime}\right),\left(i_{1}^{\prime}, \ldots, i_{n}^{\prime}\right)$ satisfying $\left(i_{1}^{\prime}, \ldots, i_{n}^{\prime}\right) \notin\left\{\left(j_{\sigma(1)}^{\prime}, \ldots, j_{\sigma(n)}^{\prime}\right): \sigma\right.$ is a permutation on $\left.\{1, \ldots, n\}\right\}$. Then $T \notin \operatorname{sm} B_{\mathcal{L}_{s}\left({ }^{n} l_{1}\right)}$.

Proof. Let $f_{1}, f_{2} \in \mathcal{L}_{s}\left({ }^{n} l_{1}\right)^{*}$ be defined by (5). Then

$$
f_{1} \neq f_{2}, f_{j}(T)=1=\left\|f_{j}\right\|(j=1,2) .
$$

Therefore, $T \notin \operatorname{sm} B_{\mathcal{L}_{s}\left({ }^{n} l_{1}\right)}$.

Acknowledgements. The author is thankful to the referees for careful reading and for suggestions that led to a better presented paper.

\section{References}

[1] W. V. Cavalcante, D. M. Pellegrino, and E. V. Teixeira, Geometry of multilinear forms, Commun. Contemp. Math. 22 (2) (2020), 1950011, 26 pp.

[2] Y. S. Choi, H. Ki, and S. G. Kim, Extreme polynomials and multilinear forms on $l_{1}$, J. Math. Anal. Appl. 228 (1998), 467-482.

[3] Y. S. Choi and S. G. Kim, Extreme polynomials on $c_{0}$, Indian J. Pure Appl. Math. 29 (1998), 983-989.

[4] Y. S. Choi and S. G. Kim, The unit ball of $\mathcal{P}\left({ }^{2} l_{2}^{2}\right)$, Arch. Math. (Basel) 71 (1998), $472-480$.

[5] Y. S. Choi and S. G. Kim, Smooth points of the unit ball of the space $\mathcal{P}\left({ }^{2} l_{1}\right)$, Results Math. 36 (1999), 26-33. 
[6] S. Dineen, Complex Analysis on Infinite Dimensional Spaces, Springer-Verlag, London, 1999.

[7] B. C. Grecu, Geometry of 2-homogeneous polynomials on $l_{p}$ spaces, $1<p<\infty$, J. Math. Anal. Appl. 273 (2002), 262-282.

[8] S. G. Kim, Exposed 2-homogeneous polynomials on $\mathcal{P}\left({ }^{2} l_{p}^{2}\right)(1 \leq p \leq \infty)$, Math. Proc. R. Ir. Acad. 107 (2007), 123-129.

[9] S. G. Kim, The unit ball of $\mathcal{L}_{s}\left({ }^{2} l_{\infty}^{2}\right)$, Extracta Math. 24 (2009), 17-29.

[10] S. G. Kim, The unit ball of $\mathcal{P}\left({ }^{2} d_{*}(1, w)^{2}\right)$, Math. Proc. R. Ir. Acad. 111 (2) (2011), 79-94.

[11] S. G. Kim, Extreme bilinear forms of $\mathcal{L}\left({ }^{2} d_{*}(1, w)^{2}\right)$, Kyungpook Math. J. 53 (2013), 625-638.

[12] S. G. Kim, Smooth polynomials of $\mathcal{P}\left({ }^{2} d_{*}(1, w)^{2}\right)$, Math. Proc. R. Ir. Acad. 113A (1) (2013), 45-58

[13] S. G. Kim, The unit ball of $\mathcal{L}_{s}\left({ }^{2} d_{*}(1, w)^{2}\right)$, Kyungpook Math. J. 53 (2013), 295-306.

[14] S. G. Kim, Exposed symmetric bilinear forms of $\mathcal{L}_{s}\left({ }^{2} d_{*}(1, w)^{2}\right)$ Kyungpook Math. J. 54 (2014), 341-347.

[15] S. G. Kim, Exposed bilinear forms of $\mathcal{L}\left({ }^{2} d_{*}(1, w)^{2}\right)$, Kyungpook Math. J. 55 (2015), 119-126.

[16] S. G. Kim, Exposed 2-homogeneous polynomials on the two-dimensional real predual of Lorentz sequence space, Mediterr. J. Math. 13 (2016), 2827-2839.

[17] S. G. Kim, Extreme 2-homogeneous polynomials on the plane with a hexagonal norm and applications to the polarization and unconditional constants, Studia Sci. Math. Hungar. 54 (2017), 362-393.

[18] S. G. Kim, The geometry of $\mathcal{L}_{s}\left({ }^{3} l_{\infty}^{2}\right)$, Commun. Korean Math. Soc. 32 (2017), 991997.

[19] S. G. Kim, The unit ball of $\mathcal{L}_{s}\left({ }^{2} l_{\infty}^{3}\right)$, Comment. Math. (Prace Mat.) 57 (2017), 1-7.

[20] S. G. Kim, Extreme bilinear forms on $\mathbb{R}^{n}$ with the supremum norm, Period. Math. Hungar. 77 (2018), 274-290.

[21] S. G. Kim, Exposed polynomials of $\mathcal{P}\left({ }^{2} \mathbb{R}_{h\left(\frac{1}{2}\right)}^{2}\right)$, Extracta Math. 33 (2) (2018), 127-143.

[22] S. G. Kim, The unit ball of the space of bilinear forms on $\mathbb{R}^{3}$ with the supremum norm, Commun. Korean Math. Soc. 34 (2019), 487-494.

[23] S. G. Kim, Extreme and exposed points of $\mathcal{L}\left({ }^{n} l_{\infty}^{2}\right)$ and $\mathcal{L}_{s}\left({ }^{n} l_{\infty}^{2}\right)$, Extracta Math. 34 (2) (2020), 127-135.

[24] S. G. Kim, Extreme points of the space $\mathcal{L}\left({ }^{2} l_{\infty}\right)$, Commun. Korean Math. Soc. 35 (3) (2020), 799-807.

[25] S. G. Kim, Smooth points of $\mathcal{L}_{s}\left({ }^{n} l_{\infty}^{2}\right)$, Bull. Korean Math. Soc. 57 (2) (2020), 443-447.

[26] S. G. Kim and S. H. Lee, Exposed 2-homogeneous polynomials on Hilbert spaces, Proc. Amer. Math. Soc. 131 (2003), 449-453.

Department of Mathematics, Kyungpook National University, Daegu 702 701, South Korea

E-mail address: sgk317@knu.ac.kr 